\title{
Thai Psychiatric Nurses' Experiences and Perceptions of the Professional Role When Caring for Older People Displaying Depressive Symptoms
}

\author{
Duangkaew Kleebthong ${ }^{1,2 *}$, Sukjai Chareonsuk ${ }^{2}$, Lisbeth Kristiansen ${ }^{1,3}$ \\ ${ }^{1}$ Deparment of Nursing Sciences, Faculty of Human Sciences, Mid Sweden University, Sundsvall, Sweden \\ ${ }^{2}$ Boromarajonani College of Nursing, Chakriraj, Thailand \\ ${ }^{3}$ Department of Health and Caring Sciences, Faculty of Health and Occupational Studies, University of Gavle, Gavle, Sweden \\ Email: *duangkaew.kleebthong@miun.se
}

How to cite this paper: Kleebthong, D., Chareonsuk, S. and Kristiansen, L. (2018) Thai Psychiatric Nurses' Experiences and Perceptions of the Professional Role When Caring for Older People Displaying Depressive Symptoms. Open Journal of Nursing, 8, 45-59.

https://doi.org/10.4236/ojn.2018.81004

Received: November 23, 2017

Accepted: January 23, 2018

Published: January 26, 2018

Copyright ( 92018 by authors and Scientific Research Publishing Inc. This work is licensed under the Creative Commons Attribution International License (CC BY 4.0).

http://creativecommons.org/licenses/by/4.0/

(c) (i) Open Access

\begin{abstract}
Purpose: The aim of this study was to describe Thai psychiatric nurses' experiences and perceptions of their professional role when caring for older people whom displayed symptoms of clinical depression. Methods: A qualitative descriptive research design was used. Thirteen psychiatric nurses working in the field of mental health and psychiatric nursing at a rural district hospital were purposively included. The data were collected through in-depth, face to face interviews and analyzed using qualitative latent content analysis. Results: All participants were female, and the mean age was 43 years, and the mean experience of caring for mentally ill patients was 8.3 years. The psychiatric nurses' experiences and perceptions of their professional role were mirrored in the following themes: 1) managing a central role in the care of the patients; 2) conflicting interests between the professional needs of caregiving and other requests; and 3) being compassionate beyond the profession. Conclusion: The psychiatric nurses were challenged by the complexity of the caregiving situations and the partners involved in the process of caring for older people with depression. Professionally, the psychiatric nurses played an important role in preventive care and managerial work. They underwent stress under the influence of stakeholders, but they also contributed to the holistic patient care. Health service authorities may use these results to develop plans for psychiatric nurses in managing a central role and in advocating for holistic care to mitigate the influence of stakeholders to enhance quality of caring for older individuals with depression.
\end{abstract}




\section{Keywords}

Depressive Disorder, Nurses, Older People, Professional Role, Thailand

\section{Introduction}

Depressive disorders are common mental health problems and may be disabling among the general older population [1]. It is estimated to become the second most common cause of disease burden by 2020 [2]. At least one in ten aged 65 or older has significant symptoms of clinical depression [3]. The prevalence of depressive disorders in the general population has been estimated by numerous epidemiological studies and surveys around the world. Previous studies showed the prevalence of depression in participants from 60 countries in all regions of the world was 3.2\% [4]. But, studies concerning the prevalence of depression in Eastern Europe countries range from $6.55 \%$ to $8.15 \%$ [5]. While, another study found the prevalence of depression was $7.4 \%$ in Japan [6]. In Thailand, the one-month prevalence of major depressive episode among older people who live in long term facilities was $23 \%$ [7].

Depression in older people in rural Thailand was experienced as living in detachment with an unwillingness to approach the health and with meaninglessness [8]. This affected both physical and psychological aspects of their lives. There was a significant high level of co-morbidity between depression and somatic illnesses such as diabetes mellitus, chronic sinusitis, back problems, and asthma [9]. Moreover, patients who suffered from depression were also faced with $22 \%$ more major adverse cardiovascular symptoms than the patients who did not have depression [10]. Further, depression is positively related to interference with human functioning, deterioration of health, thought, and memory problems, persistent feelings of sadness, and hopelessness [11]. Physicians may be more likely to explore physical symptoms than psychological symptoms in older patients. Additionally, older people may be less likely to report symptoms of depression and may be less comfortable discussing emotions than younger generations [12]. It is, thus, a misunderstanding that depression is a normal condition of the older people that develops with age or physical illnesses [12]. This idea has often led to misdiagnosis and has prevented patients with depressive disorders from receiving prompt treatment [13]. Above all, depression is strongly related to suicidal ideation, which can lead to suicide among older people [14].

Many Thai individuals interpret their illness according to Buddhist's construction of Karma and reincarnation [15]. This means that if a person has behaved morally bad in a previous life, he would suffer in his current life but by practicing karmic healing activities may end the suffering, and ensure a better life next time, and therefore, there is no need to medically treat the illness in present life [15]. Besides, Thai families are considered to be mainly an extended 
family-type that regarded it as a moral obligation to care for ill relative [16]. This might even be the case regarding depression. In general, nurses are in an individual position to assess patients for depression [17]. In a same way, Thai registered nurses screen individuals respectively with 2 -symptom screenings (2Q) and patient health questionnaires-9 (9Q), especially high-risk individuals, such as patients with chronic illness, addiction, and older people in their communities for depressive symptoms at least once a year. However, depressive disorders are seldom addressed in mental healthcare service [18]. In Thailand, patients with depression in primary healthcare are treated by general practitioners because there is a lack of psychiatrists [19]. Moreover, the relapse rate in a depressed Thai population $(\mathrm{n}=224)$ was almost $39 \%$ [20], which indicated that Thai mental healthcare system has a potential to be developed regarding the treatment of depression.

\section{Theoretical framework}

In general, psychiatric nurses had an important professional role as they were specialized in communication and the establishment of interpersonal relationships [21] with patients suffering from psychiatric illness. This paper utilized Peplau's understanding of interpersonal relationship establishment [21]. In addition, in Thai rural areas, psychiatric nurses now work comprehensively at the forefront of health care delivery due to a lack of psychiatrists.

\section{Rationale}

Taken together depression is of big concern also in a Thai context. There are huge challenges in the Thai mental and psychiatric health care. Some patients do not gain access to proper health care, and even some of those patients who get adequate care and treatment, still showed high levels of relapses. Besides, there is a lacking knowledge on how the psychiatric nurses themselves experience the work and their professional role in the rural areas in the provinces in Thailand. Given the central part in the mental and psychiatric health care, this study sought to provide an understanding of how Thai psychiatric nurses described their experiences and their perceptions of the professional role when caring for older people in the rural area whom displayed symptoms of depression.

\section{Methods}

\subsection{Study Design}

To capture the variations in Thai psychiatric nurses' experiences, a qualitative research design with an inductive approach inspired by Graneheim and Lundman [22] and Patton [23], was utilized to describe the latent content in these. This approach ontologically acknowledged the existence of multi realities [24]. Further, the study also investigated the perceptions of the professional role.

\subsection{Research Context and Participants}

The study was conducted in the rural areas of Kanchanaburi province. It is about 129 kilometers away from Bangkok, with a borderline to Burma. The total area is 
about 19,473 square kilometers. The province had 848, 198 inhabitants. The setting was under the secondary care geographical division of public health in Thailand.

The participants were recruited from a sample of registered nurses who were also specialized in psychiatric and mental health nursing in public health settings in Kanchanaburi province, and they were purposively selected to suite the research objective. The inclusion criteria were: working in the field of mental health and psychiatric nursing at a district hospital, and having more than two years of experience in caring for older people with a depressive disorder. The nurses were provided with study details and information about informed consent. This was done to ensure that they understood the research thoroughly before independently deciding to participate. Thirteen Thai nurses agreed to participate in qualitative interviews. All participants were women. A general information about participants were provided in Table 1.

\subsection{Data Collection}

This study used an in-depth interview method. Every participant was contacted and an appointment at a convenient time was made and, further, they agreed to digital recording during the interviews. Each face-to-face interview session took approximately 45 - 90 minutes, and all were conducted between April and October 2015. Open-ended questions were posed to provide an opportunity for the informants to casually tell their stories. To address to study aim, the interview questions were concentrated in the following two domains:

1) Please, tell me about your professional role as a psychiatric nurse at the district hospital.

2) Please, tell me about your experiences when working with older people who display symptoms of major depressive disorder.

Table 1. Characteristics of Psychiatric Nurses Participated.

\begin{tabular}{|c|c|c|c|c|}
\hline Number & Age (year) & status & Education & Experience working as a psychiatric nurses (year) \\
\hline 1 & 40 & Single & MS & 5 \\
\hline 2 & 44 & Single & MS & 10 \\
\hline 3 & 34 & Single & MS & 5 \\
\hline 4 & 37 & Married & MS & 5 \\
\hline 5 & 40 & Married & MS & 9 \\
\hline 6 & 38 & Married & MS & 8 \\
\hline 7 & 43 & Married & MS & 9 \\
\hline 8 & 42 & Single & MS & 5 \\
\hline 9 & 42 & Single & BS & 5 \\
\hline 10 & 51 & Married & MS & 13 \\
\hline 11 & 50 & Single & MS & 10 \\
\hline 12 & 52 & Married & BS & 15 \\
\hline 13 & 50 & Single & BS & 10 \\
\hline
\end{tabular}


Clarifying questions and follow-up questions were asked to provide richer data. All interviews were verbatim transcribed and saved in the form of word document files (Approximately160 pages).

\subsection{Data Analysis}

The interview data were analyzed using latent content analysis inspired by Graneheim and Lundman [22] and Patton [23]; the process is explained in detail in the following section.

First, the transcriptions were repeatedly scrutinized to thoroughly comprehend the content. Second, in relation to the study aim, meaning units were identified, which was words, sentences, and paragraphs with key meaning. Third, the meaning units were condensed while keeping the original essence of the text. Forth, the condensed meaning units were abstracted and labeled with codes. With the principle of similarities and differences, the various codes were itemized into subthemes related to the objectives of the study. Finally, the subthemes were then compared, sorted and abstracted into themes. An example of the analysis process is in Table 2, and Table 3 shows an overview of subthemes and themes.

Table 2. Example of analysis process.

I couldn't screen for depression in all the older people in this district alone. There are thousands of them, it was impossible. I then have to teach the method of screening elders with $2 \mathrm{Q}$ to the other general nurses who are responsible for other chronic disease departments, such as the diabetes or

Meaning units

Condensed meaning unit

Coding

Subthemes

Themes hypertension clinics. I also have to depend on other networks, such as the nurses at the primary health care centers. I have to teach them how to do the basic screening so they can help pre-screen the risk group and send them to me for further screening with $9 \mathrm{Q}$. After that, I would check the elder's score, evaluate, record the data in the computer, and send that elder to see a physician.

Screening for depression with a large number of older people could not be done completely by one psychiatric nurse so the method of screening was taught to nurses to screen the older people. The primary screening was confirmed and recorded by psychiatric nurse after they were sent to a physician.

Screening for depression

Being responsible for patient screening procedures.

Managing a central role in the care of the patients.

Table 3. Overview of subthemes and themes.

\begin{tabular}{|c|c|}
\hline Subthemes & Themes \\
\hline $\begin{array}{l}\text { Being responsible for patient screening procedures. } \\
\text { Acting in a life-saving manner. }\end{array}$ & Managing a central role in the care of the patients. \\
\hline $\begin{array}{l}\text { Dealing with pressure due to expectations from various } \\
\text { stakeholders. }\end{array}$ & Conflicting interests between the professional need of caregiving and other requests. \\
\hline Advocating for holistic care of the patients. & \\
\hline Feeling of relative bond. & Being compassionate beyond the profession \\
\hline Honor of acting as a savior for the patient. & \\
\hline $\begin{array}{l}\text { Frustration due to imbalance between stakeholder } \\
\text { demands and nurse resources. }\end{array}$ & \\
\hline
\end{tabular}




\section{Trustworthiness}

To address credibility, the researchers constantly reviewed and reevaluated the analysis, confirming that relevant data were included, and that irrelevant data cautiously excluded. During the whole process, the research team discussed the meaning units, including condensed meaning units, coding, subthemes, themes, and critical analysis, to enhance credibility. The research team worked to obtain consensus. To address dependability, moreover, the first author interviewed three of 13 participants twice to conduct a member checking. The research team also confirmed the recognition of the result.

\subsection{Ethical Considerations}

This study was approved by the research ethics committee (Ethical no.: 1-15-2558), and the procedures were in accordance with the Declaration of Helsinki. Participants were informed about the purpose of this study, their voluntary participation, and that they could withdraw from the study at any time without any consequence. The information was confidential and the results were published for the benefit of academic purposes only. After the participants agreed to participate in the research, they signed the informed consent form.

\section{Findings}

The psychiatric nurses were challenged by the complexity of the caregiving situation and by the partners involved in the process of caring for older people with affective symptoms or illnesses. The results are based on the abstraction of three themes: managing a central role in the care of the patients, conflicting interests between the professional needs of caregiving and other requests, and being compassionate beyond the profession. The three themes are described in more detail below. All of the participants in the study, $100.0 \%$ were female, the mean age was 43.3 years, $53.84 \%$ were single, $76.92 \%$ graduated master degree, and the mean experiences of caring for mentally ill patients was 8.3 years.

\subsection{Managing a Central Role in the Care of the Patients}

This theme was related to how the psychiatric nurses performed professionally toward patients with affective illnesses or symptoms. The psychiatric nurses played an important role in preventive care, as well as in managerial work. This theme was based on the abstraction of the content in the following two subthemes: being responsible for screening procedures, and acting in a life-saving manner.

\subsubsection{Being Responsible for Patient Screening Procedures}

The psychiatric nurses were responsible for performing all screening procedures to detect depressive symptoms in the entire community of individuals over 60 years of age. For this purpose, they used two validated questionnaires (2Q) (1: Have you been felt down, depressed, or hopeless during the last two weeks? and 2: Have you been bored with life, or lost interest or pleasure in doing things 
during the last two weeks?). If there was a positive result, patient health questionnaires-9 (9Q) (e.g., Over the last 2 weeks, how often have you felt tired or had little energy? How often have you had thoughts that you would be better off dead, or of hurting yourself in some way?), were confirmedly administered by the nurse. Depending on the scores, four possible actions were taken: no action, mild, moderate, or severe. As the population was large, approximately 5000 older individuals per psychiatric nurse, the psychiatric nurses had to educate and supervise registered nurses to assist in the procedure. This is an example of one statement from an informant.

"I couldn't screen all the older people for depression in this district alone. There are thousands of them, it was impossible. I, then, have to teach the method of screening older with $2 Q$ to other general nurses who are responsible for other chronic disease departments, such as the diabetes mellitus or hypertension clinics. I also have to depend on other networks, such as the nurses at the primary health care centers. I have to teach them how to do the basic screening so they can help pre-screen risk groups and send them to me for further screening with 9Q. After that, I would check the older people's scores, evaluate, record the data in the computer, and refer the older person to a physician." (Participant 11)

\subsubsection{Acting in a Life-Saving Manner}

This subtheme was related to findings regarding the psychiatric nurses' experiences of operating in a life-saving manner. Most psychiatric nurses stated that besides common symptoms, such as insomnia, headache, dizziness, or various aches, they saw co-morbidity related challenges in the patients. This could, for instance, consist of uncontrolled diabetes mellitus, often leading to hyperglycemia or hypoglycemia, metabolic syndrome, or uncontrolled or poorly medicated hypertension. This is exemplified by the following citation.

"One old lady who got diabetes and used to receive medicine every two months, as usual, she faced the problem of being unable to control her glucose and regularly had hyperglycemia. The physician at OPD referred her to me. I opened some casual conversation with her for a while, and gradually asked questions in order to find out some reasons for her problems while screening for her depression at the same time. The scores showed that she was depressed. I gave her psycho-educational information and reported the results to the physician. The physician then prescribed anti-depression medicine to this patient. After that, this patient was getting better and her glucose was lower."(Participant 2)

\subsection{Conflicting Interests between the Professional Needs of Caregiving and Other Requests}

This theme was related to the stress the psychiatric nurses experienced under the influence of stakeholders and while contributing to the holistic care for the patients. This theme was based on the abstraction of the content in the following 
two subthemes: dealing with pressure due to expectations from various stakeholders, and advocating for holistic care of the patients.

\subsubsection{Dealing with Pressure Due to Expectations from Various Stakeholders}

To provide care for older people with depression, the psychiatric nurses had to address many expectations from both the health care team and patients or their relatives. The health care team, including the general practitioners and registered nurses, requested that the psychiatric nurses advised them on the difficulties related to mental health issues. The general practitioners also consulted with the psychiatric nurses to confirm the treatment plans.

"A physician consulted me about a patient with tuberculosis who refused to take medicine regularly. The physician was afraid that his patient may have a drug-resistant version of $T B$ and the inflection would be spread through her body. In this case, I had to visit the patient at home to identify the causes of her problem. It appeared that she lived with her husband and a little grandchild. Her children lived next door, but they seemed to be separated; they didn't care about and didn't talk to each other. Her husband told me that she was lazy; she didn't do anything except sit still and hug her own knees. After I had screened this patient with $9 Q$, I found that she suffered from a severe depression and suicidal ideations. I then explained the symptoms of depression and tuberculosis to her husband so he could help take care of her regular medicine, and I wrote a report to her physician for further treatment." (Participant 3 )

The patients and their relatives often asked for reassurance from the psychiatric nurses. Frequently, the psychiatric nurses found that the patients returned to the hospital with complaints about problems related to their quality of life and their daily lives, for instance, the inability to sleep, feelings of being uncomfortable, and feelings of being under stress or in relapse. When the psychiatric nurses examined, that there were generally no serious health problems that required professional medical help. However, the psychiatric nurses sensed that the patients were still suffering and wanted nursing care. The psychiatric nurses were frustrated because they could not fulfill this nursing duty due to their limited resources.

"Working in a psychiatric clinic, besides giving counseling to my patients, $I$ have to counsel with other medical personnel in multiple disciplines, such as physicians, nurses from OPD or IPD, and even the non-serious patients who have already been discharged from the clinic, but needed to see me again. I couldn't take care all of them alone since I am the only psychiatric nurse in this hospital." (Participant 5)

\subsubsection{Advocating for Holistic Care of the Patients}

When the psychiatric nurses engaged in home visits, which were a part of their job, they often identified problems related to unmet physical, social, and finan- 
cial needs. Although, these problems did not need direct nursing care, having a holistic mindset caused the nurses to be concerned, and they felt morally obligated to take actions on behalf of the patient.

"While I visited a patient who was a 78-year-old female, with no children and who lost her husband long ago, she hardly walked and moved; she lived in an old house that was close to collapsing, only accompanied by a distant relative. I felt sad for her and decided to report her case to the Provincial Administrative Organization and the Kanchanaburi Provincial Social Development and Human Security Office. The officers from both departments came to visit and help the patient. Now, she has a new small house. I also contacted volunteers in the community for elderly service, which is the group that encourages members in the community to help or take care of each other. I feel happy to see all of them." (Participant 10)

\subsection{Being Compassionate beyond the Profession}

This theme was based on the abstraction of the content in the following three subthemes: feeling of relative bond, honor of acting as a savior for the patient, and frustration due to imbalance between stakeholder demands and nurse resources.

\subsubsection{Feeling of Relative Bond}

While caring for older individuals with depression, the psychiatric nurses felt close to the patients. They referred to the patients as they were their uncles or aunts, and provided comfort as they were with familiar people by touching them during the counseling process, such as holding their hands or gentle patting their arms. They sat closely to the patients and casually talked to them about general topics to gain their trust. The psychiatric nurses also monitored the condition of their patients by visiting them at their homes outside of work hours. Moreover, the patients also showed affection towards the nurses. They treated the nurses like they would their own relatives. This is example of one statement from an informant.

"When I saw depressed older people come to the hospital, I would view them as my own uncles or aunts because they were the same age. I would feel sorry for them because, in some cases, the older people came to the hospital alone, without any company. I would casually persuade them to talk and make them feel that the nurse is not furious. If a nurse looks nice, a patient would have the courage to talk." (Participant 12)

Moreover, it is found that the psychiatric nurses spent their time monitoring their cases at home because of their own personal concerns. As reported by one nurse:

"I met a patient who was still sitting at the hospital after she received medicine. I asked her why she was still here, and she said that she waited for her neighbors to pick her up after they finished their business in another place. I kept asking her about her life and knew that she lived with her husband, who was also older, 
and another two grandchildren that their parents left for her to take care of while they were working in another province. I really felt for her that she had to bare so many burdens about her grandchildren, even though she already had her own troubles. I then told her that if it was inconvenient to come to the hospital, I would visit her at home instead." (Participant 11)

\subsubsection{Honor of Acting as a Savior for the Patient}

The older individuals with depression were constantly in need of medicine. They were treated with anti-depressants on a daily base, and depending on the severity of depression, the psychiatrics nurses evaluated the status every two weeks or every month. Here the psychiatric nurses continuously noticed changes and patient progressions starting from severe depression to recovery. The psychiatric nurses were proud to be a part of a remedy for the patients, as reported by one nurse.

"I met a depressed patient and started taking care of her since the beginning. When she first visited the hospital, she repeatedly said that she wanted to die or she did not want to live anymore, but after getting treatments, she was better. Her face became fresh, like normal people again. I was happy that she felt better and proud to bring her back to normal." (Participant 9)

Furthermore, the psychiatric nurses were deeply proud they had the qualifications and competences to being able to caring properly for the patients with depression. For example, the patients confidentially told the psychiatric nurses more about their background stories than they told the registered nurses.

"There was a patient admitted to the ward two or three days ago that the registered nurses in the ward tried to ask about her own conditions, but she did not talk to any nurse. She only slept motionless on her bed, closing her eyes. I was requested by her physician to evaluate her condition. I started establishing a relationship with the female patient, when she trusted me, she began to share her inner feelings with me. I was proud that I could make her talk to me, despite the fact that she slept badly, and that she did not talking to anyone for several days." (Participant 3)

\subsubsection{Frustration Due to Imbalance between Stakeholder Demands and Nurse Resources}

The policy of the district hospitals required psychiatric nurses to respond not only to the psychiatric and mental health of the patients, and to other special groups of patients, such as drug addicted, abused children and women, but also to conduct mental health consultation sin multidisciplinary teamwork. This made the psychiatric nurses carry too many burdens. Sometimes, these burdens included emergency cases that were sent from other wards concurrently; such a deteriorated situation can be exemplified from one of psychiatric nurse with the following citation:

"On some very busy days, I would like to split myself into many parts so I could do more work in less time. One day, while I consulted a patient, a nurse from ward called me to talk with another patient who was having suicide idea- 
tions. I started to worry and did not know what to do. While, I was treating the patient in front of me, I was also concerned about the other."(Participant 6 )

\section{Discussion}

The aim of this study was to describe Thai psychiatric nurses' experiences and perceptions of their professional role when caring for older people that displayed symptoms of depression. The findings are based on the abstraction of three themes: managing a central role in the care of the patients, conflicting interests between the professional need of caregiving and other requests, and being compassionate beyond the profession.

\subsection{Managing a Central Role in the Care of the Patients}

Until now it has been unknown that in the rural Thailand it seems that it is the psychiatric nurses, who are mainly responsible for screening older people for depression. The older have often been referred to the psychiatric nurse from general practitioner or the general nurse. As older individuals with depression frequently do not receive appropriate diagnosis and treatment [25], and as they often display somatization instead for "clear" symptoms of depression, the symptoms can be difficult to interpret as depression for general practitioners and registered nurses [26]; thus, psychiatric nurses play a central role. Some patients believe that their illness is caused by an act in a previous life, according to Buddhist beliefs [15]. Therefore, Thai medical practices or health care services must bear this in mind, when they interact with and provide the patients with proper diagnosis and treatment of depression [20]. If not diagnosed or properly treated, depression can increase the risk of suicide, as there is a close relationship between depression and suicidal ideation [14].

Among patients with chronic diseases such as chronic obstructive pulmonary disease, kidney disease, Parkinson's disease, cardiovascular disorders, and diabetes mellitus were positively associated with depression [27]. With the professional role of psychiatric nurses, the sooner the depression is treated, the healthier the patient will be in various aspects. For instance, the general practitioner found that although a patient with diabetes mellitus was treated continuously, the blood sugar level was abnormal. After that, the general practitioner referred the patient to the psychiatric nurse. She established a relationship with the patient, screened with $9 \mathrm{Q}$, counseled the patient, and reported back to the general practitioner. The general practitioner appropriately treated the condition and, consequently, the patient completely recovered. If psychiatric nurses could evaluate and provide proper treatment for depression, they could help save the lives of their patients. This is the important role, the psychiatric nurses often identified that these referred patients had symptoms of depression when they evaluated with $9 \mathrm{Q}$, the results showed that more than half of the patients suffered from depression, which is consistent with a prior study showing that physical illness usually occurs with depression [5] [27]. According to psychiatric nurses 
they have found depression in patients with co-morbidities. This professional knowledge may lead to that these patients get the proper treatment.

\subsection{Conflicting Interests between the Professional Needs of Caregiving and Other Requests}

According to the result, there is only one psychiatric nurse in the district hospital who takes care of older mental health and psychiatric patients. For this reason, psychiatric nurses had high expectation placed upon them from the stakeholders such as multidisciplinary and patients and their family. The results showed that the psychiatric nurse needs to take holistic care of the patients, and when the patients have problems or need help, the psychiatric nurse usually contacts related departments to acquire help for the patients. When caring for patients with mental health problems, the relationship between a nurse and a patient is the most important factor that affects the outcome of health care [28]. This nurse-patient relationship has a special paradigm in nursing. The process begins with the nurse establishing a relationship with the patient based on trust. After the working phase, in which the patient works with the nurse to solve problems, the nurse determines that the patient has improved and he/she enters the termination phase [21], which means the patient returns to normal life without depending on the nurses. However, the results showed that patients and their relatives still needed help from the nurses, even though the patients could be discharged from the clinic. One reason is because depression has a strong chance of recurring in older people [29]. The nurses need to be alert and sensitive the patients' needs. Together with others' expectations, it may cause nurses to experience mental health problems on their own bodies as they are in conflict with the nurse need of recovery to stay professional, as Tavares et al. [30] found that professional nurses faced minor psychiatric disorders $20.1 \%$. With consist, the three main problems leading of affirmative answers were experiencing nervousness, tension or worry (49.2\%), poor sleeping (39.2\%), and tiring easily (37.7\%).

\subsection{Being Compassionate beyond the Profession}

The psychiatric nurses in this study were open-minded and friendly towards the patients. They treated patients as if they were their own relatives. As a result, the psychiatric nurses were proud and happy to see the patients getting better after their treatment. This is in line with the theory and practice of mental health and psychiatric nursing, nurses not only engage in therapeutic works, but they also have to concern about the humanity of their patients, as they are unique individuals. When nurses are sincere and friendly towards patients, friendship arises, which fosters successful treatments [31]. Conversely, if they could not appropriately care for the patients, the nurses were worried, frustrated, as shown in our results. These emotions can lead to burnout. The prior research found that higher psychiatric nurse-to-patient staffing ratios are one of the factors that cause psychiatric nurse burnout [32]. Moreover, it also found that an increased workload or lack of resources are the main stressors of the psychiatric nurses. 
Everyone has been compassionate at some point during their career. Sometimes, these feelings are positive, and other times, they are negative, depending on the workload or resource supply. Therefore, psychiatric nurses are also compassionate beyond the profession.

\section{Limitations of study}

There were 13 psychiatric nurses obtained purposively for this research. All participants work in the district hospital. However, these findings do not present the only interpretation of the experiences and perceptions, but via the presentation of citations, we tried to make the reader hear the informants' voices. Given the detailed description of the research circumstance, the reader is enabled to decide if the result may be transferred into psychiatric nurse experiences in other district hospitals in Thailand, or elsewhere.

\section{Conclusion}

This study explored psychiatric nurse experiences and professional roles when caring for older people with depression, and found that psychiatric nurses play an important role in caring for psychiatric patients. They may provide psychosocial interventions, nurse-directed services in healthcare contexts, and psychiatric nursing consultation services. Moreover, their experiences in caring for older people with depression explained the challenges involved in managing and responding to various expectations in complex situations. The results from this study provide introductory data that may be used by health service authorities to develop plans for psychiatric nurses to enhance the quality of care for older people with depression, though more research is needed to understand these aspects.

\section{Acknowledgements}

The authors would like to acknowledge all the psychiatric nurses in Kanchanaburi who were involved in the study. We would also like to thank Associate professor in nursing K. G. Norbergh for his advice during the manuscript phase. This study was supported financially by Mid Sweden University, Sweden, and Boromarajonani College of Nursing, Chakriraj, Thailand.

\section{Conflicts of Interest}

The researchers report no conflict of interest.

\section{References}

[1] Verhaak, P.F., Dekker, J.H., de Waal, M.W., van Marwijk, H.W. and Comijs, H.C. (2014) Depression, Disability and Somatic Diseases among Elderly. Journal of Affective Disorders, 167, 187-191. https://doi.org/10.1016/j.jad.2014.05.057

[2] Murray, C.J.L. and Lopez, A.D. (1996) The Global Burden of Disease. Harvard University Press, Boston.

[3] Manthorpe, J. and Iliffe, S. (2005) Depression in Late Life. Jessica Kingsley, London.

[4] Moussavi, S., Chatterji, S., Verdes, E., Tandon, A., Patel, V. and Ustun, B. (2007) 
Depression, Chronic Diseases, and Decrements in Health: Results from the World Health Surveys. Lancet, 370, 851-858. https://doi.org/10.1016/S0140-6736(07)61415-9

[5] Van de Velde, S., Bracke, P. and Levecque, K. (2010) Gender Differences in Depression in 23 European Countries. Cross-National Variation in the Gender Gap in Depression. Social Science \& Medicine, 71, 305-313. https://doi.org/10.1016/j.socscimed.2010.03.035

[6] Inagaki, M., Ohtsuki, T., Yonemoto, N., Kawashima, Y., Saitoh, A., Oikawa, Y., et al. (2013) Validity of the Patient Health Questionnaire (PHQ)-9 and PHQ-2 in General Internal Medicine Primary Care at a Japanese Rural Hospital: A Cross-Sectional Study. General Hospital Psychiatry, 35, 592-597.

https://doi.org/10.1016/j.genhosppsych.2013.08.001

[7] Wongpakaran, N. and Wongpakaran, T. (2012) Prevalence of Major Depressive Disorders and Suicide in Long-Term Care Facilities: A Report from Northern Thailand. Psychogeriatrics, 12, 11. https://doi.org/10.1111/j.1479-8301.2011.00383.x

[8] Kleebthong, D., Chareonsuk, S. and Kristiansen, L. (2017) Older Thai Peoples' Experiences of Major Depression. Global Journal of Health Science, 9, e-Version First TM. ISSN 1916-9736 E- ISSN 1916-9744.

[9] Gunn, J.M., Ayton, D.R., Densley, K., Pallant, J.F., Chondros, P., Herrman, H.E., et al. (2010) The Association between Chronic Illness, Multimorbidity and Depressive Symptoms in an Australian Primary Care Cohort. Social Psychiatry and Psychiatric Epidemiology, 47, 175-184. https://doi.org/10.1007/s00127-010-0330-z

[10] Jani, B.D., Cavanagh, J., Barry, S.J.E., Der, G., Sattar, N. and Mair, F.S. (2016) Relationship between Blood Pressure Values, Depressive Symptoms, and Cardiovascular Outcomes in Patients with Cardiometabolic Disease. The Journal of Clinical Hypertension, 18, 1027-1035. https://doi.org/10.1111/jch.12813

[11] Townsend, M. (2008) Essentials of Psychiatric Mental Health Nursing: Concepts of Care in Evidenced-Based Practice. 4th Edition, F. A. Davis, Philadelphia.

[12] Bryant, C. (2010) Anxiety and Depression in Old Age: Challenges in Recognition and Diagnosis. International Psychogeriatrics, 22, 511-513.

https://doi.org/10.1017/S1041610209991785

[13] Monteso, P., Ferre, C., Lleixa, M., Albacar, N., Aguilar, C., Sanchez, A., et al. (2012) Depression in the Elderly: Study in a Rural City in Southern Catalonia. Journal of Psychiatric and Mental Health Nursing, 19, 426-429. https://doi.org/10.1111/j.1365-2850.2011.01798.x

[14] Shin, K.M., Cho, S.M., Hong, C.H., Park, K.S., Shin, Y.M., Lim, K.Y., et al. (2013) Suicide among the Elderly and Associated Factors in South Korea. Aging and Mental Health, 17, 109-114. https://doi.org/10.1080/13607863.2012.702732

[15] Nilmanat, K. and Street, A.F. (2014) Karmic Quest: Thai Family Caregivers Promoting a Peaceful Death for People with AIDS. Contemporary Nurse, 27, 94-103. https://doi.org/10.5172/conu.2007.27.1.94

[16] McCann, T.V., Songprakun, W. and Stephenson, J. (2015) A Randomized Controlled Trial of Guided Self-Help for Improving the Experience of Caring for Careers of Clients with Depression. Journal of Advanced Nursing, 71, 1600-1610. https://doi.org/10.1111/jan.12624

[17] Winkley, K. (2013) Depression and Diabetes: What Should Nurses Do? Nurse Times, 109, 20-22.

[18] Glogoski-Williams, C. (2008) Recognition of Depression in the Older Adult. Occu- 
pational Therapy in Mental Health, 15, 17-34.

https://doi.org/10.1300/J004v15n02_02

[19] Lotrakul, M. and Saipanish, R. (2006) Psychiatric Services in Primary Care Settings: A Survey of General Practitioners in Thailand. BMC Family Practice, 7, 48. https://doi.org/10.1186/1471-2296-7-48

[20] Hiranyatheb, T., Nakawiro, D., Wongpakaran, T., Wongpakaran, N., Bookkamana, P., Pinyopornpanish, M., et al. (2016) The Impact of Residual Symptoms on Relapse and Quality of Life among Thai Depressive Patients. Neuropsychiatric Disease and Treatment, 12, 3175-3181. https://doi.org/10.2147/NDT.S124277

[21] Peplau, H.E. (1962) Interpersonal Techniques: The Crux of Psychiatric Nursing. The American Journal of Nursing, 62, 50-54. https://doi.org/10.1097/00000446-196206000-00028

[22] Graneheim, U.H. and Lundman, B. (2004) Qualitative Content Analysis in Nursing Research: Concepts, Procedures and Measures to Achieve Trustworthiness. Nurse Education Today, 24, 105-112. https://doi.org/10.1016/j.nedt.2003.10.001

[23] Patton, M.Q. (2015) Qualitative Research \& Evaluation Methods. 4th Edition, Sage, Thousand Oaks.

[24] Creswell, J.W. (2007) Qualitative Inquiry \& Research Design-Choosing among Five Approaches. 2nd Edition, Sage, London.

[25] Gellis, Z.D. (2010) Depression Screening in Medically Ill Homecare Elderly. Best Practice Guidance for Mental Health Services, 6, 1-16.

[26] Wolpert, L. (2001) Stigma of Depression: A Personal View. British Medical Bulletin, 57, 221-224. https://doi.org/10.1093/bmb/57.1.221

[27] Peltzer, K. and Pengpid, S. (2016) Anxiety and Depressive Features in Chronic Disease Patients in Cambodia, Myanmar and Vietnam. South African Journal of Psychiatry, 22, a940. https://doi.org/10.4102/sajpsychiatry.v22i1.940

[28] Peplau, H.E. (1988) Interpersonal Relations in Nursing: A Conceptual Frame of Reference for Psychodynamic Nursing. 2nd Edition, Macmillan Education, Basingstoke. https://doi.org/10.1007/978-1-349-10109-2

[29] Comijs, H.C., Nieuwesteeg, J., Kok, R., van Marwijk, H.W., van der Mast, R.C., Naarding, P., et al. (2015) The Two-Year Course of Late-Life Depression: Results from the Netherlands Study of Depression in Older Persons. BMC Psychiatry, 15, 1-9. https://doi.org/10.1186/s12888-015-0401-5

[30] Tavares, J.P., Magnago, T.S.B.S., Beck, C.L.C., Silva, R.M., Prestes, F.C. and Lautert, L. (2014) Prevalence of Minor Psychiatric Disorders in Nursing Professors. Escola Anna Nery Revista de Enfermagem, 18, 407-414. https://doi.org/10.5935/1414-8145.20140058

[31] Arnold, E. and Underman-Boggs, K. (2011) Interpersonal Relationships: Professional Communication Skills for Nurses. 6th Edition, Elsevier Saunders, St. Louis.

[32] Hanrahan, N.P., Aiken, L.H., McClaine, L. and Hanlon, A.L. (2010) Relationship between Psychiatric Nurse Work Environments and Nurse Burnout in Acute Care General Hospitals. Issues in Mental Health Nursing, 31, 198-207. https://doi.org/10.3109/01612840903200068 